\title{
An interactive method of fuzzy probability elicitation in risk analysis
}

\author{
E. Vicente \\ Decision Analysis and Statistics Group, Universidad Politécnica de Madrid, Spain
}

\author{
A. Jiménez \\ Decision Analysis and Statistics Group, Universidad Politécnica de Madrid, Spain \\ A. Mateos \\ Decision Analysis and Statistics Group, Universidad Politécnica de Madrid, Spain
}

\begin{abstract}
Expert knowledge is used to assign probabilities to events in many risk analysis models. However, experts sometimes find it hard to provide specific values for these probabilities, preferring to express vague or imprecise terms that are mapped using a previously defined fuzzy number scale. The rigidity of these scales generates bias in the probability elicitation process and does not allow experts to adequately express their probabilistic judgments. We present an interactive method for extracting a fuzzy number from experts that represents their probabilistic judgments for a given event, along with a quality measure of the probabilistic judgments, useful in a final information filtering and analysis sensitivity process.
\end{abstract}

\section{INTRODUCTION}

The subjective approach is often the only option for assigning probabilities of event occurrence about which no previous experience or data are available. Abbas et al. (2008), Cojazzi et al. (1987), Morgan \& Henrion (1990) and Stäel Von Holstein \& Matheson (1979), among others, have proposed different methods for assigning subjective probabilities. These methods enact indirect bias avoidance processes (Tversky \& Kahaneman, 1974) during expert probability assignment

The best known of these processes are elicitation methods based on betting and lotteries by Savage (1954) and Finetti (1964). These methods are recommended by the most influential subjective probability elicitation protocols, such as SRI (Stanford Research Institute), SNL/NUREG-1150 (U.S. Nuclear Regulatory Commission) and KEEJAM (Joint Research Center of the European Commission).

However, experts may find it hard to assign these probabilities using real numbers, and the use of linguistic terms, such as "low", "high" or "medium", is a more feasible option. We can then associate fuzzy numbers with the linguistic terms, which we can use in the computations throughout risk analysis.

Normalized trapezoidal fuzzy numbers with support in [0,1], i.e. the tuples $\tilde{A}=\left(a_{1}, a_{2}, a_{3}, a_{4}\right)$, with $0 \leq a_{1} \leq a_{2} \leq a_{3} \leq a_{4} \leq 1$, whose membership function has a trapezoidal shape, are a set of fuzzy numbers that are especially useful because of the simplicity of the arithmetic operations defined on them (Chen 1985, Chen \& Chen 2007). In particular, if $a_{2}=a_{3}$, then the number is called triangular, and if $a_{1}=a_{2}$ and $a_{3}=a_{4}$, the number is rectangular, and denotes a crisp interval in $[0,1]$. Moreover, real numbers $a \in[0,1]$ are injected into the fuzzy numbers through $a \approx$ $\widetilde{a}=(a, a, a, a) \in T F[0,1]$, where $T F[0,1]$ denotes the set of normalized trapezoidal fuzzy numbers with support in $[0,1]$.

The arithmetic operations for trapezoidal fuzzy numbers are reported in Chen (1985), Chen \& Chen (2007) and Xu et al. (2010). We denote these arithmetic operations by (+), (-), ( $\times$ ) and (/).

These numbers have been adapted for use in many mathematical risk analysis models in an imprecise or vague context. Dokas et al. (2007) give good examples of fault trees adapted to the 
fuzzy paradigm. For instance, for the fault tree shown in Figure 1, the experts are asked to use the scale by Dokas et al. (see Table 1 and Fig. 1) to assign linguistic terms to the probabilities of the basic events BE1, BE2 and BE3. The probability for the top event (TE) is computed on the basis of these assignments by means of the operation associated with the logic gates (Dokas et al., 2007).

Table 1. Two possible fuzzy linguistic term scales

\begin{tabular}{lcc}
\hline Term & Scale by Dokas et al. & Alternative scale \\
\hline Very Low & $(0,0,0.1,0.2)$ & $(0,0,0,0.25)$ \\
Low & $(0.1,0.2,0.2,0.3)$ & $(0,0.05,0.15,0.25)$ \\
Fairly Low & $(0.2,0.3,0.4,0.5)$ & $(0.15,0.25,0.35,0.45)$ \\
Medium & $(0.4,0.5,0.5,0.6)$ & $(0.35,0.45,0.55,0.65)$ \\
Fairly High & $(0.5,0.6,0.7,0.8)$ & $(0.55,0.65,0.75,0.85)$ \\
High & $(0.7,0.8,0.8,0.9)$ & $(0.75,0.85,0.95,1)$ \\
Very High & $(0.8,0.9,1,1)$ & $(0.95,1,1,1)$ \\
\hline
\end{tabular}
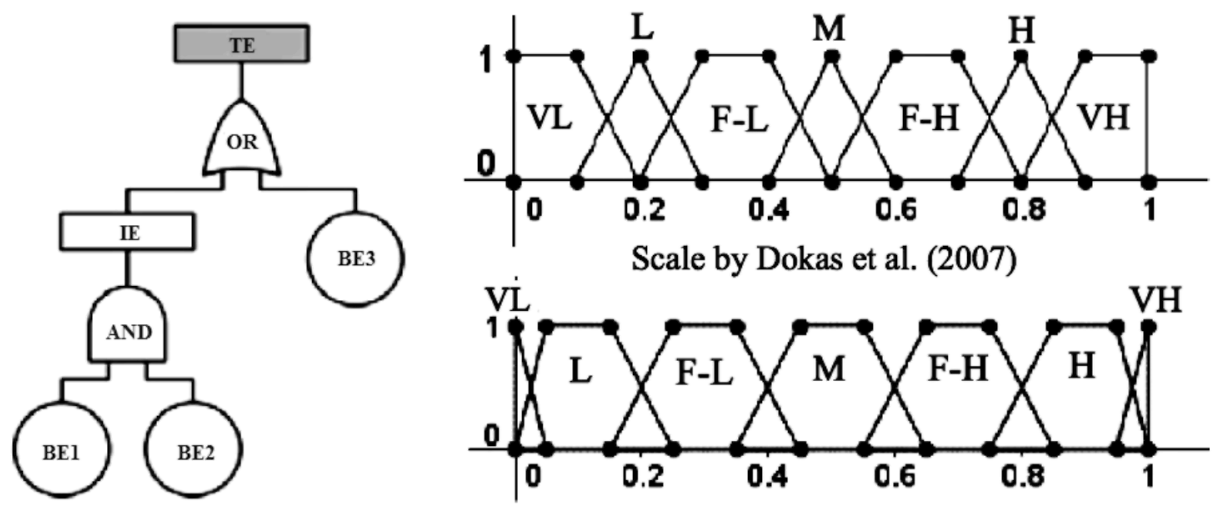

Alternative scale

Figure 1. Dokas et al.'s fault tree example and two fuzzy linguistic term scales.

The example is solved in Dokas et al. (2007) by assigning probabilities $\mathrm{H}, \mathrm{M}$ and $\mathrm{L}$ to the basic events BE1, BE2 and BE3, respectively. The solution derived for the TE is the probability represented by the fuzzy number $(0.35,0.52,0.52,0.68)$.

However, if we use the alternative scale (see Table 1 and Fig. 1), the result is then $(0.26$, $0.41,0.59,0.73$ ). Consequently, the result can differ widely depending on the scale we use even if both scales represent the same linguistic term set.

Assigning a fuzzy probability $(a, b, c, d)$ to an event means that the expert is sure that the probability is in the interval $(a, d)$, and is more likely to be within $[b, c]$; this likelihood decreases linearly from $b$ to $a$ and from $c$ to $d$.

The expert has no say about the trapezoidal fuzzy numbers that are fixed to the linguistic term set. However, the appearance of the fuzzy numbers could differ depending on what knowledge the expert has about the event. It would be worthwhile, for example, considering more or less accurate or symmetrical fuzzy numbers, but the rigidity of the fixed scale does not account for such information. In other words, not all scales are suitable for all experts and for all events.

On the other hand, the direct assignment of probabilities can be significantly biased with respect to the circumstances in which the assignment process is developed. The use of direct scales for assignment is very simplistic.

Throughout this paper we develop a method for extracting a fuzzy number representing a probabilistic judgment for each event from experts, in order to avoid biases possibly generated by previously established rigid scales.

In Section 2 we briefly overview fuzzy probability theory, which extends classical probability theory. In Section 3, we develop an interactive method for fuzzy probability elicitation. Section 4 provides measures to analyze the quality of the information provided by the expert. These 
measures will be useful for filtering the information subject to a minimum quality requirement, and are helpful for performing a sensitivity analysis in a fuzzy environment. Finally, Section 5 gives an illustrative example.

\section{FUZZY PROBABILITY THEORY}

Fuzzy probability theory was developed by Helliwell (2007). Fuzzy numbers with support in $[0,1]$ and, in particular, trapezoidal fuzzy numbers denoted by $T F[0,1]$, extend the real numbers in $[0,1]$ and constitute a complete metric space whose distance is the maximum Hausdorff distance for the $\alpha$-cuts.

The completeness of $T F[0,1]$ allows the definition of convergent sequences and series and is useful for defining the fuzzy probability of the countable union of pairwise disjoint events as the sum of the fuzzy probabilities of each event, that is, the completeness of $T F[0,1]$ extends the third axiom of Kolmogorov to trapezoidal fuzzy numbers. Formally, a fuzzy probability space is a triple $\left(\Omega, H, p^{\gamma}\right)$, where $\Omega$ is the event space of a random experiment, $\mathrm{H}$ is an $\sigma$-algebra of $\Omega$, and $p^{f}: \mathrm{H} \rightarrow T F[0,1]$ is a function that verifies the following axioms:

-Axiom 1: $\widetilde{0} \leq p^{f}(\widetilde{E}) \leq \widetilde{1}, \forall \widetilde{E} \in T F[0,1]$. The order between fuzzy probabilities is defined in Helliwell (2007).

-Axiom 2: $p^{f}(\Omega)=\widetilde{1}$.

-Axiom 3: Given a countable family of pairwise disjoint events $E_{i} \in \mathrm{H}$, then $p^{f}\left(\bigcup_{i} E_{i}\right) \subseteq(+)_{i} p^{f}\left(E_{i}\right)$, where $\subseteq$ is the subsumption relation in $T F[0,1]$.

-Axiom 4: $p^{f}\left(E^{c}\right)=\widetilde{1}(-) p^{f}(E), \forall E \in \mathrm{H}$.

Note that the first three axioms naturally extend to the three classical Kolmogorov axioms. However, we have need of the fourth axiom because fuzzy arithmetic operations have no inverse element, and therefore the complementary probability cannot be assessed by fuzzy algebraic equations from the first three axioms, as in classical probability theory.

To elicit the probability function of a random discrete variable, we have to define a complete set of pairwise disjoint events, $\left\{E_{i}\right\}_{i=1}^{n}$, and assign a probability to each event $\tilde{\pi}_{i}=P^{f}\left(E_{i}\right)$, so that $\tilde{\pi}_{i} \subseteq \tilde{1}(-)\left[(+)_{j \neq i} \tilde{\pi}_{j}\right]$. Otherwise, there is no guarantee that the Kolmogorov axioms will hold in probability elicitation (Helliwell 2007). Risk analysis usually requires this type of variables since most randomized experiments are dichotomous, such as "failure" or "no failure".

\section{FUZZY PROBABILITY ELICITATION BASED ON BETTING AND LOTTERIES}

The betting and lottery-based methods commonly used to assign probabilities can also be used to assign fuzzy probabilities (Savage, 1954; Finetti, 1964). In this section we briefly describe these methods and show how a fuzzy number representing the probability judgment can be extracted from experts.

Betting Method. For two selected monetary values $x>y$, the expert is given the option of participating in one of the two following games:

(bl) If event $A$ happens, then you win $x €$. Otherwise, you lose $y €$.

(b2) If event $A$ does not happen, then you win $y €$. Otherwise, you lose $x €$.

If the expert has no preference for either bet, the respective expected utilities of both bets are equal, and it follows that $p(A)=x /(x+y)$. If the expert chooses one of the two games, then the expected utility of the selected game should be higher than for the rejected game. Then, the analyst has to update monetary values and offer to the expert two new games. Thus, an interactive process is enacted until two alternative games are reached to which the expert is indifferent.

Lottery-based methods. For a given probability $p \in[0,1]$ and monetary values $x €$ and $y €$, the following lotteries the expert is given the choice between the: 
(l1) If event $A$ happens, then you win $x €$. Otherwise, you lose $y €$.

(l2) You win $x €$ with probability $p$, or $y €$ with probability $1-p$.

If expert has no preference for either of the lotteries, then the respective expected utilities are equal, and it follows that $p(A)=p$. Otherwise, the expert must readjust the value $p$, keeping the same monetary values. This again generates an interactive process, enacted until a couple of lotteries are reached to which the expert is indifferent.

The betting and lottery-based methods assume that the expert is able to provide a specific value for the probability of an event. However, a more realistic scenario is where experts have an imprecise and vague idea of that value. Consequently, experts will have an interval rather than a precise value in mind at the point when they are indifferent to either bet or lottery, that is, for the lottery-based method there will be an interval $[a, c]$ such that if $p[a, c]$, then expert has no preference for either lottery $(l 1)$ or $(l 2)$. Similarly, the betting method can result in an interval of indifference $[b, d]$.

Current protocols for probability elicitation like the above recommend the use of several methods to test the consistency of the expert and the existence of bias. In this regard, the development of betting and lottery-based methods meets this recommendation and establishes the following:

- If $[a, c] \cap[b, d]=\varnothing$, then the expert's probabilistic judgment was inconsistent.

- If any of the intervals is contained in the other $[a, c] \subseteq[b, d]$ (or $[b, d] \subseteq[a, c]$ ), then we assume that the trapezoidal fuzzy number $(b, a, c, d)$ (or $(a, b, d, c)$ ) designates the expert probabilistic judgment.

- If $[a, c] \cap[b, d] \neq \varnothing$, is uncountable, and none of the intervals is contained in the other, then, assuming that $a \leq b \leq d \leq c,(a, b, d, c)$ designates the expert probabilistic judgment.

To elicit the probability function of random discrete variables, commonly used in risk analysis, we must as pointed out in Section 2 assign the probabilities for each event from a complete system of pairwise disjoint events verifying that $\tilde{\pi}_{i} \subseteq \widetilde{1}(-)\left[(+)_{j \neq i} \tilde{\pi}_{j}\right]$, in order to assure that fuzzy Kolmogorov axioms hold.

\section{QUALITY OF PROBABILISTIC JUDGMENTS. ACCURACY AND CONSISTENCY MEASURES}

As pointed out in Section 3, if $[a, c] \cap[b, d]=\emptyset$, then the expert has been inconsistent in the elicitation process. However, if the intersection is a single point, $c=b=\S$, then the situation is practically identical, and it leads to the triangular fuzzy number $(a, \xi, d)$. Then, a triangular number represents the limit between consistency and inconsistency in probabilistic judgments from betting and lottery-based methods.

On the other hand, if $[a, c]=[b, d]$, the expert has been totally consistent, and the fuzzy number representing the probabilistic judgment is a rectangular fuzzy number, i.e. a crisp interval. Thus, the shape of the fuzzy number (triangular, trapezoidal or rectangular) reflects the consistency of the expert judgment, which can be measured by

$$
C(a, b, c, d)=\left\{\begin{array}{cc}
\frac{c-b}{d-a} & \text { if } d-a \neq 0 \\
1 & \text { if } d-a=0
\end{array}\right.
$$

This measure verifies that

- $C(\widetilde{A}) \in[0,1], \forall \widetilde{A} \in T F[0,1]$.

- $C(\widetilde{A})=1 \Leftrightarrow \Leftrightarrow \widetilde{A}$ is a crisp number, and

- $C(\tilde{A})=0 \Leftrightarrow \Leftrightarrow \tilde{A}$ is triangular.

$C$ is a continuous function in the complete metric space $T F[0,1]$, and is invariant under any fuzzy operation by scalars, i.e. for all real numbers $\widetilde{\lambda}=(\lambda, \lambda, \lambda, \lambda)$ and for any fuzzy operation $\left.(*), C\left(\lambda^{*}\right) \tilde{A}\right)=\tilde{C}(\tilde{A})$.

Besides the consistency demonstrated by the expert, we might be interested in the accuracy of his/her probabilistic judgment. An expert that opts for a fuzzy probability of $(0.2,0.22,0.23$, 
$0.25)$ using betting and lottery-based methods is much more accurate than an expert that assigns a fuzzy probability of $(0,0,1,1)$. A higher accuracy indicates that experts are more sure about their probabilistic judgment.

We can measure the accuracy accounting for the area under the membership function of the trapezoidal fuzzy number. The function $S(a, b, c, d)=[c-b+d-a] / 2$ indicates the accuracy of the expert's probabilistic judgment. This function verifies $S(\tilde{A}) \in[0,1], \forall \widetilde{A} \in T F[0,1]$, where $S(\tilde{A})$ $=1 \Leftrightarrow \Leftrightarrow \tilde{A}$ is a real number, and $S(\tilde{A})=0 \Leftrightarrow \Leftrightarrow \tilde{A}$ is the interval $[0,1]$. $S$ is a continuous function in the complete metric space $T F[0,1]$, and $\tilde{A} \subseteq \tilde{B} \Rightarrow S(\tilde{A}) \geq S(\tilde{B})$.

Finally, the quality of the probabilistic judgment expressed by the expert can be assessed by aggregating the previous functions, $Q(\widetilde{A})=C(\widetilde{A}) \times S(\tilde{A}), \forall \widetilde{A} \in T F[0,1]$. This function is also continuous in $T F[0,1], Q(\tilde{A}) \in[0,1], \forall \widetilde{A} \in T F[0,1]$, becomes 0 when the fuzzy number is triangular or the interval $[0,1]$, and is $l$ if and only if the number is a real number.

This quality measure filters the information given by the expert and determines how the system would improve if there were crisp rather fuzzy values on the probability of an event.

Let us assume that an expert has assigned probabilities to the basic events BE1, BE2 and BE3 in the fault tree shown in Figure 1 using the betting and lottery-based methods as a result of an interactive process. Suppose that we have obtained the trapezoidal fuzzy numbers $(0.75,0.8$, $0.85,0.9),(0.35,0.4,0.5,0.62)$ and $(0.1,0.4,0.5,0.5)$, respectively.

The quality of these probabilistic judgments is $0.29,0.3$ and 0.18 respectively. This demonstrates the flexibility of the proposed method by means of which experts can state more or less precise probabilistic judgments. This is not possible if we use the rigid scales introduced in Section 1. Accordingly to these values, the result of the fault tree for the TE is the trapezoidal fuzzy number $(0.33,0.59,0.71,0.77)$, and the associated quality is 0.19 .

Now, it would be interesting to analyze how the quality might improve if the expert were to provide a crisp rather than a fuzzy number for the probability of any basic event. To do this, we can repeat the analysis by entering the value in the system and comparing the new quality values output.

As pointed out in Section 4, expert consistency is invariant under any operation using crisp numbers. However, the accuracy of the probabilistic judgment is invariant to the sum of crisp numbers but sensitive to the product of a crisp number, since $S(\lambda, \lambda, \lambda, \lambda)(\times)(a, b, c, d)=1-[\lambda(c$ $-b+d-a)] / 2$, which depends on the value of $\lambda$. Therefore, if the expert provides a crisp value for the probability of the event, that crisp value will influence the system quality. The quality will differ depending on which real numbers are considered.

We propose using the real number $\lambda \approx \tilde{\lambda}$ that maximizes $\operatorname{Sim}(\tilde{\lambda}, \tilde{A})$ to get an idea of the quality improvement that the use of a crisp value would mean, where Sim is a similarity function for fuzzy numbers and $\tilde{A}$ is the fuzzy number initially provided by the expert in the probabilistic judgment.

In most similarity functions described in literature, such as Vicente et al. (2013a;b), Chen \& Chen (2007) and Xu et al. (2010), the real value that maximizes the similarity to a given fuzzy number is the abscissa of the geometric center of the similarity function, i.e. $x=y\left(a_{3}+a_{2}\right)+(1$ $-y)\left(a_{4}+a_{1}\right)$, where $y=\left(a_{3}-a_{2}\right) /\left[6\left(a_{4}-a_{1}\right)\right]+1 / 3$.

For example, if the expert changes his/her fuzzy probabilistic judgment for the basic event BE3, $(0.1,0.4,0.5,0.5)$, for a crisp number given by its center of gravity, $(0.35,0.35,0.35$, $0.35)$, the probability for TE would now be $(0.52,0.56,0.62,0.71)$, with a quality of 0.3 , which would improve the quality of the result with respect to the original fuzzy judgment by over $50 \%$. 


\section{CONCLUSIONS AND FUTURE WORK}

We have developed a fuzzy probability elicitation process that is flexible enough to properly determine the accuracy and consistency that each expert can provide for each event, along with a quality measure that is used to filter information and run a qualitative sensitivity analysis. This process is useful in fuzzy risk analysis, where it outperforms the traditional models that establish rigid fuzzy scales for probability elicitation.

In future work, we intend to develop a process for simulating analyst-expert dialogue in order to control probability elicitation biases and check the effect of such biases on the results of the process. A real case will be used to illustrate the methodology.

\section{ACKNOWLEDGMENT}

This paper was supported by the Madrid Regional Government project S-0505/TIC/0230 and the Spanish Ministry of Science and Innovation project MTM2011-28983-C03-03.

\section{REFERENCES}

Abbas, A.E., Budescu, D.V., Hsiu-Ting, Y. \& Haggerty, R. 2008. A Comparison of two probability encoding methods: fixed probability vs. fixed variable values. Decision Analysis 5(4): 190-202.

Chen, S.M. (1985). Operations on fuzzy numbers with function principle. Tamkang Journal of Management Science 6: 13-25.

Chen, S.J. \& Chen S.M. 2007. Fuzzy risk analysis based on the ranking of generalized trapezoidal fuzzy numbers. Intelligent Applications 26: 1-11.

Cojazzi, G., Guida, G., Pinola, L., Sardella, R. \& Baroni, P. 1987. KEEJAM: a knowledge engineering methodology for expert judgement acquisition and modelling in probabilistic safety assessment. In C. Guedes Soares (ed.), Advances in Safety and Reliability 1: 199-206. New York: Pergamon.

Dokas, I.M., Nordlander, T.E. \& Wallace, R.J. 2007. Fuzzy fault tree representation and maintenance based on frames and constraint technologies: a case study. In Proc. K-CAP'07 Workshop on Knowledge Capture and Constraint Programming, Whistler, British Columbia, Canada.

Finetti, B. 1964. Foresight: its logical laws, its subjective sources. In H.E. Kyburg and H.E. Smokler (eds.), Studies in Subjective Probability. New York: Wiley.

Halliwell, J. 2007. Linguistic Probability Theory. PhD Thesis. Edinburgh: University of Edinburgh.

Morgan, M.G. \& Henrion, M. 1990. Uncertainty: A guide to dealing with Uncertainty in Quantitative Risk and Policy Analysis. Cambridge: Cambridge University Press.

Savage, L.J. 1954. The Fundations of Statistics. New York: Wiley.

Stäel Von Holstein, C.A. \& Matheson, H.A. 1979. Manual For Encoding Probability Distributions. SRI International. Stanford: Stanford Research Institute.

Tversky, A. \& Kahneman, D. 1974. Judgment under uncertainty: heuristics and biases. Science, New Series 185(4157): 1124-1131

Vicente, E., Jiménez, A. \& Mateos, A. 2013a. A fuzzy approach to risk analysis in information systems. In Proc. 2nd intern. conf. on operations research and enterprise systems, Barcelona 16-18 February 2013.

Vicente, E., Mateos, A. \& Jiménez, A. 2013b. A new similarity function for generalized trapezoidal fuzzy numbers. In Proc. 12th intern. Conf. on Artificial Intelligence \& Soft Computing, Zakopane 9-13 June 2013. To appear.

Xu, Z., Shang, S., Qian, W. \& Shu, W. 2010. A method or fuzzy risk analysis based on the new similarity of trapezoidal fuzzy numbers. Expert Systems and Applications 37: 1920-1927. 